\title{
Algorithm Performance Investigation of a Generalized Spreader-Bar Detection System
}

SM Robinson ED Ashbaker WK Hensley JE Schweppe GA Sandness LE Erikson JH Ely

October 2010

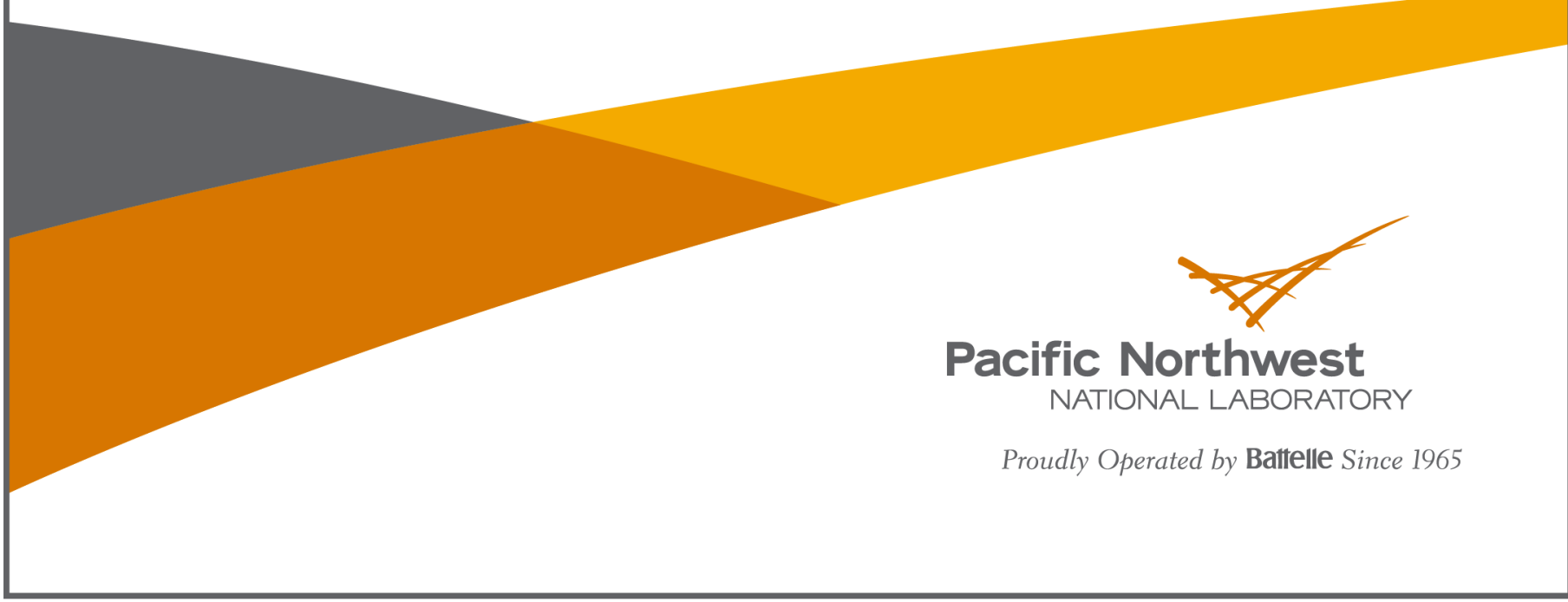




\title{
DISCLAIMER
}

This report was prepared as an account of work sponsored by an agency of the United States Government. Neither the United States Government nor any agency thereof, nor Battelle Memorial Institute, nor any of their employees, makes any warranty, express or implied, or assumes any legal liability or responsibility for the accuracy, completeness, or usefulness of any information, apparatus, product, or process disclosed, or represents that its use would not infringe privately owned rights. Reference herein to any specific commercial product, process, or service by trade name, trademark, manufacturer, or otherwise does not necessarily constitute or imply its endorsement, recommendation, or favoring by the United States Government or any agency thereof, or Battelle Memorial Institute. The views and opinions of authors expressed herein do not necessarily state or reflect those of the United States Government or any agency thereof.

\author{
PACIFIC NORTHWEST NATIONAL LABORATORY \\ operated by \\ BATTELLE \\ for the \\ UNITED STATES DEPARTMENT OF ENERGY \\ under Contract DE-AC05-76RL01830
}

Printed in the United States of America
Available to DOE and DOE contractors from the Office of Scientific and Technical Information,
P.O. Box 62, Oak Ridge, TN 37831-0062;
ph: (865) 576-8401
fax: $(865)$ 576-5728
email: reports@adonis.osti.gov

\begin{abstract}
Available to the public from the National Technical Information Service, U.S. Department of Commerce, 5285 Port Royal Rd., Springfield, VA 22161 ph: (800) 553-6847 fax: $(703) 605-6900$ email: orders@ntis.fedworld.gov online ordering: http://www.ntis.gov/ordering.htm
\end{abstract}

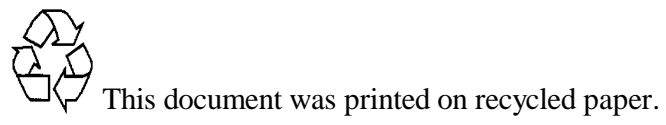




\section{Algorithm Performance Investigation of a Generalized Spreader-Bar Detection System}

SM Robinson

ED Ashbaker

WK Hensley

JE Schweppe

GA Sandness

LE Erikson

JH Ely

September 2010

Pacific Northwest National Laboratory

Richland, Washington 99352 


\section{Executive Summary}

A "generic" gantry-crane-mounted spreader bar detector has been simulated with the Monte-Carlo radiation transport code MCNP [1]. This model is intended to represent the largest feasible number of detector crystals in a single gantry-crane model intended to sit atop an intermodal cargo container (IMCC). We chose detectors from among large and commonly available sodium iodide crystal scintillators, and spaced them as evenly as possible within a detector apparatus attached to a gantry crane.

Several scenarios were simulated with this model, which was based on a single IMCC to be moved between a ship's deck or cargo hold and the dock. During measurement, a gantry crane carries an IMCC through the air and lowers it onto a receiving vehicle (e.g., chassis or bomb cart). The case of an IMCC being moved through the air from an unknown radiological environment to the ground is somewhat complex; for this initial study, we chose a single location at which to simulate background. A highly enriched uranium source, based on earlier validated models, was used and placed at varying depths in a wood cargo. Many statistical realizations of these scenarios are constructed from simulations of the component spectra, simulated to have high statistics. To produce these results, we have only considered this single HEU source and statistical realizations of the background; we simulated no NORM or other nuisance sources. The resultant data have been analyzed with several different algorithms, and are intended to provide a representative set of the analysis techniques which might be chosen for a real detector:

- Gross Counting: The gross-counting algorithm is one of the simplest methods of source detection, and is used in many deployed detector schemes. In this algorithm, the number of counts above the background is computed and compared to a threshold. The figure of merit is generally calculated from

\section{CBIA}

where $\mathrm{C}$ is the total counts and B is the background counts. Gross counting is thought to be an effective "baseline" solution, because its performance cannot be significantly changed by anything other than attenuation by cargo materials.

- Energy Distance: In this approach, the available spectrum is binned into coarse energy bins that are treated as a vector and normalized. Then the "opening angle" between background and source spectra is found by taking the dot product between them. This opening angle is the figure of merit returned from this algorithm. Similar algorithms have been useful in identifying sources behind cargo.

- Peak Finding: The peaks present in the spectroscopic data are "picked out" with a variety of methods and then compared with a catalog of known source emission lines to produce a list of the isotopes present in the measured spectrum. The spectrum is convolved over energy with a matching filter, compared to a threshold to generate peaks, and then the peaks are compared to a limited catalog of sources to determine source detection.

- GADRAS: In the Gamma Detector Response and Analysis Software (GADRAS) template matching code, the spectrum to be interpreted is compared with a library of templates. When a "good" match is found, the spectrum can be identified as that of the isotope used to generate the template. The template library contains the measured or calculated spectra of most isotopes, as well as some background spectra. 
Template matching is well suited to the identification of unshielded radiation sources. Multiple regressions can also be applied to identify combinations of radionuclides.

- Limited GADRAS: In this scheme, only the natural isotopes expected in background are included in the template library, meaning that the presence of an additional source will tend to make the "goodness of fit" progressively worse with intensity. Thus, the goodness of fit $\left(\mathrm{Chi}^{2}\right)$ is used as the figure of merit for this use of GADRAS, rendering it an anomaly detecting algorithm.

The simulated data were evaluated by each algorithm, with a threshold set to a false alarm probability of 0.001, and the resultant minimum detectable amounts were generated for each cargo depth possible within the IMCC. In this report, we calculated the false alarm probabilities using only the statistical count variation of the background, and no nuisance sources were included in the data sets. Probabilities of Detection were generated for each cargo depth and source strength combination, and these were analyzed to produce the Minimum Detectable Amount (MDA) of the HEU source for each cargo depth, as shown below. The MDA is herein defined as the source intensity (in emitted gammas per second from the source surface) at which a 95\% Probability of Detection is reached. These data are presented with a line representing $25 \mathrm{~kg}$ of HEU (actually a 25-fold multiplier on the standard 1-kg source used for simulations) for comparison. In our evaluation of these data, the technique of using GADRAS as an anomaly detector provided the greatest detection sensitivity. I We expect that an algorithm similar to this will be greatly useful in the detection of sources shielded by substantial amounts of cargo. However, the "worst case" scenario - detection of sources shielded by several meters of dense cargo-still presents a difficult problem for even the best algorithms considered. Future work will be necessary to improve on the current algorithms and refine these results.

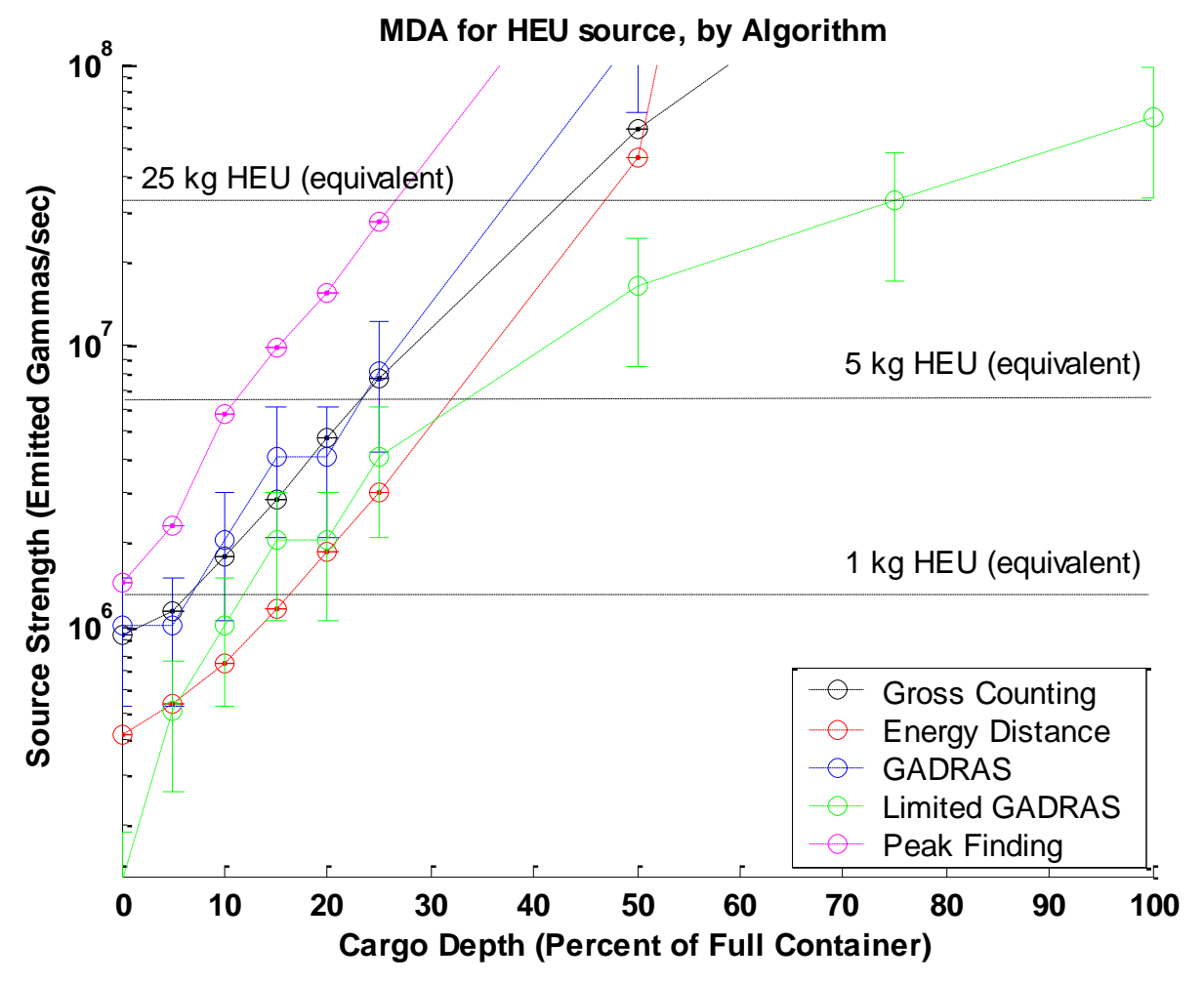

Figure ES-1. Minimum Detectable Amounts for Highly Enriched Uranium 


\section{Acronyms and Abbreviations}

cps

HEU

IMCC

MCNP

$\mathrm{NaI}$

NORM

SBRD

GADRAS counts per second

highly enriched uranium

intermodal cargo container

Monte-Carlo N-Particle

sodium iodide

naturally occurring radioactive material

spreader-bar radiation detector

Gamma Detector Response and Analysis Software 


\section{Contents}

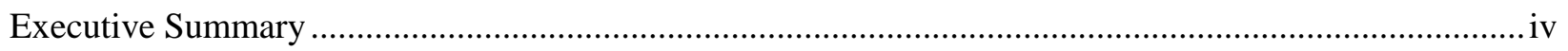

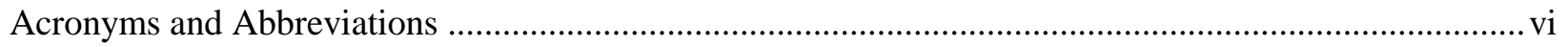

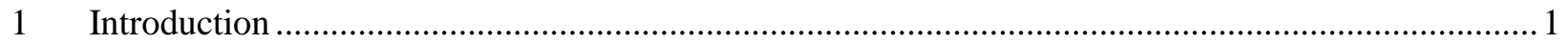

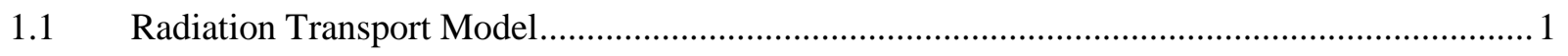

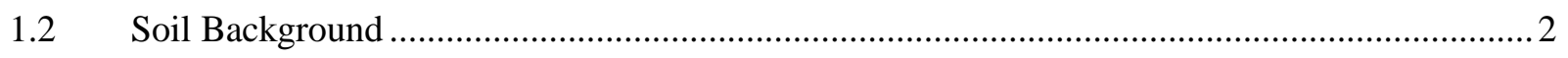

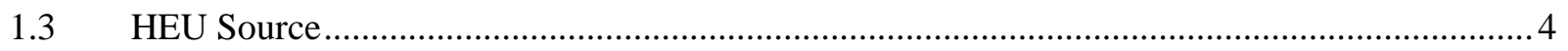

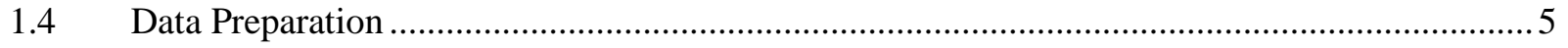

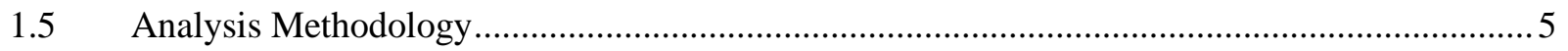

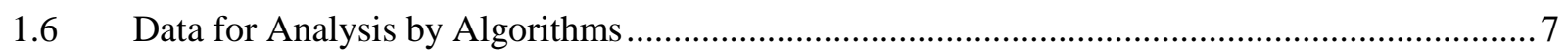

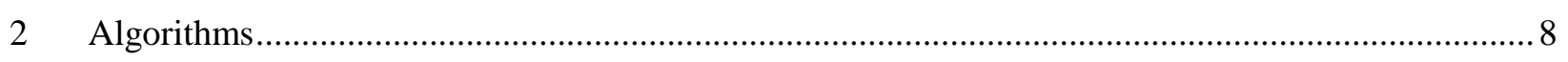

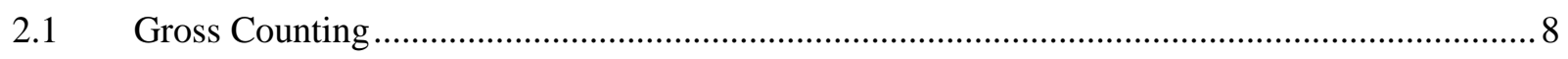

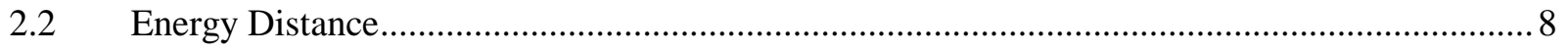

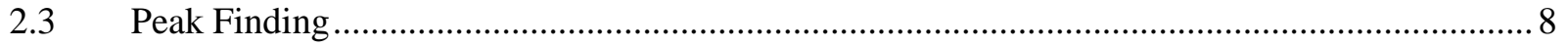

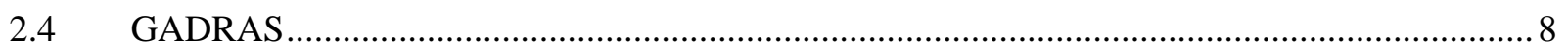

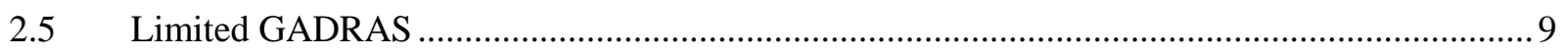

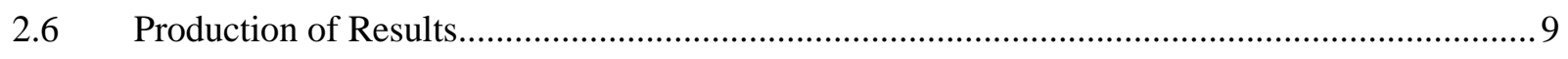

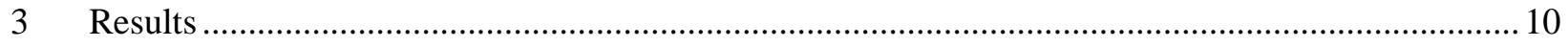

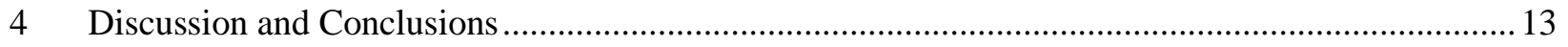

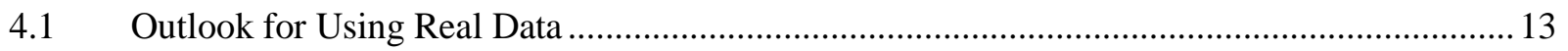

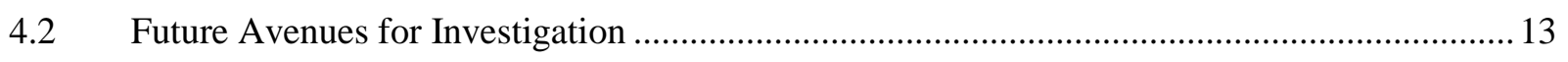

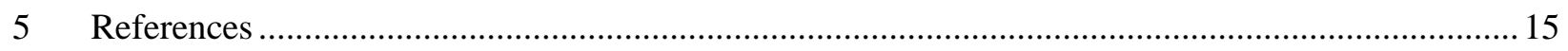




\section{Figures and Tables}

\section{Figures}

Figure 1-1. Top View of SBRD NaI Detector Crystal Locations ........................................................ 1

Figure 1-2. SBRD with NaI Detector Crystal Locations and Photomultiplier Tubes ................................2

Figure 1-3. Scenario for Background Simulations......................................................................... 2

Figure 1-4. Soil Background Detected by SBRD Central Detector ........................................................ 3

Figure 1-5. HEU Source Detected by SBRD Central Detector............................................................. 4

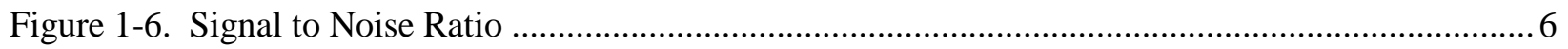

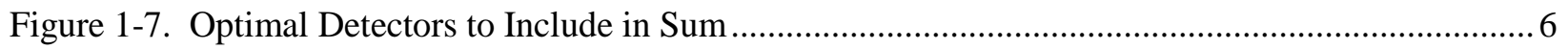

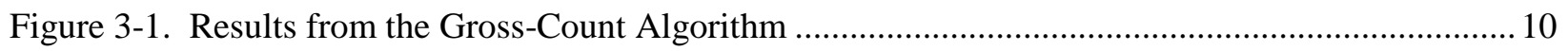

Figure 3-2. Results from the Energy Distance Algorithm .............................................................. 11

Figure 3-3. Minimum Detectable Amounts for HEU Source ............................................................ 12

\section{Tables}

Table 1-1. Soil Background Simulation Output Files ............................................................................ 3

Table 1-2. HEU Source Simulation Output Files ................................................................................ 4 


\section{Introduction}

A spreader-bar-mounted detection system was simulated to determine the baseline detection capability for shielded sources and the behavior of individual algorithms. We produced several models for background, detectors, cargo, and sources of interest, with the intent of evaluating a generalized case of source detection. These models are not meant to represent the result from any actual system currently manufactured. Several caveats should be applied to the result of this work, especially in regard to the use of a single source and cargo type. It is possible that higher-energy sources may be more easily detectable by spectroscopic methods or that denser or more high-Z cargo would make detection more difficult. However, to compare the performance of algorithms to one another, the cargo amount and source strength were significantly varied to provide a look at every potential shielding depth and realistic source strength possible within a cargo container. The spreader-bar model includes (75) $4^{\prime \prime} \times 4^{\prime \prime} \times 16^{\prime \prime}(10.16 \times 10.16 \mathrm{x}$ $40.64 \mathrm{~cm}$ ) sodium iodide (NaI) detectors distributed on or near the bottom surface of the spreader bar. This large number of detectors was incorporated to optimize source detectability by the use of detector subsets. However, in this study only a central source location was used. In all cases, scenarios were modeled to estimate the detectability of a simple, highly enriched uranium (HEU) source inside an intermodal cargo container (IMCC) and underneath varying thicknesses of wood cargo.

\subsection{Radiation Transport Model}

Elements of the spreader bar, IMCC, cargo, source, and surrounding environment were modeled in order to simulate gamma rays leaving the source, effects of the environment, and the process of detection. The "generic" gantry-crane-mounted spreader-bar-detector array described above was simulated in the Monte Carlo radiation transport code MCNP [1], and is referred to hereafter as the spreader-bar radiation detector (SBRD) model. Detectors were chosen from among large, commonly available, NaI crystal scintillators and spaced as evenly as possible. A top view of the relative positions of the detectors on the SBRD is shown in Figure 1-1. There are 20 detector locations in each of the end sections of the spreader bar and 35 detector locations in the middle section of the spreader bar (see Figure 1-2). This spatial distribution of detectors was chosen to provide a similar amount of detector coverage on each segment of an actual gantry-crane mounted spreader bar device like the ones used at seaports.

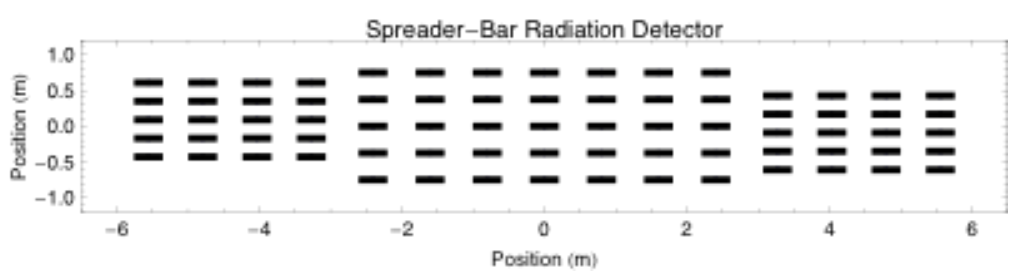

Figure 1-1. Top View of SBRD NaI Detector Crystal Locations

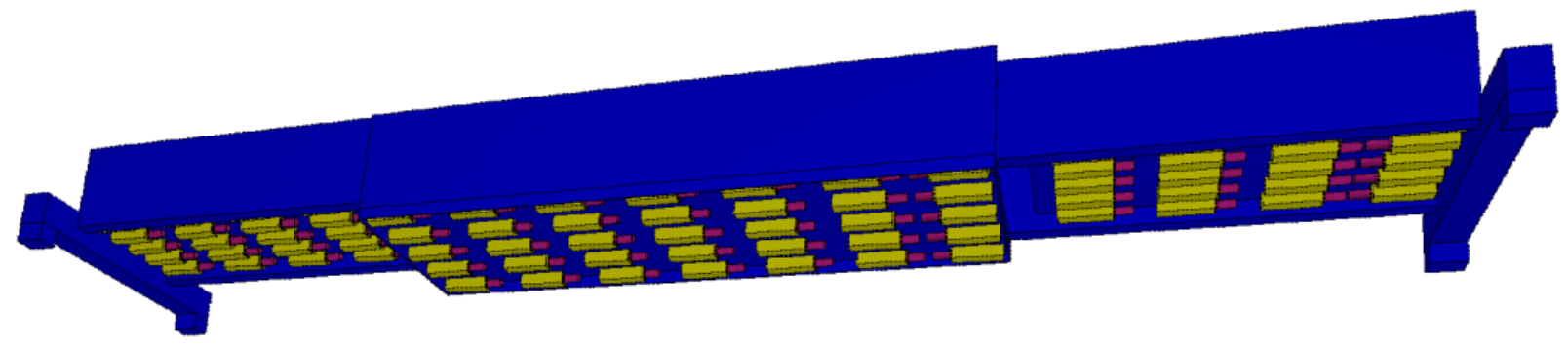




\section{Figure 1-2. SBRD with NaI Detector Crystal Locations and Photomultiplier Tubes}

During measurement, the gantry crane will lower the spreader bar onto a single IMCC within a ship's hold or on deck, carry that IMCC through the air and lower it onto a receiving vehicle (e.g., a chassis or bomb cart). The case of an IMCC being moved through the air from the unknown radiological environment of a ship to the ground is complex, so the issue of background variation during this exercise is likewise complex. In practice, we expect that any real source detection algorithm will need to exclude data taken within some reasonable distance from the ship to avoid unpredictable variations in the background due to emissions from nearby cargo containers. Additionally, the background variation between water and land may be significant. In this work, the scenario was simplified by placing the modeled container in a fixed position on the ground 6 meters from the edge of the water. Figure 1-3 shows the water, ground, IMCC, and detector from the background simulation. All simulations were made by using MCNP [1] with sufficiently small statistical uncertainty that later data manipulations could be made to generate many statistical realizations of shorter measurements.

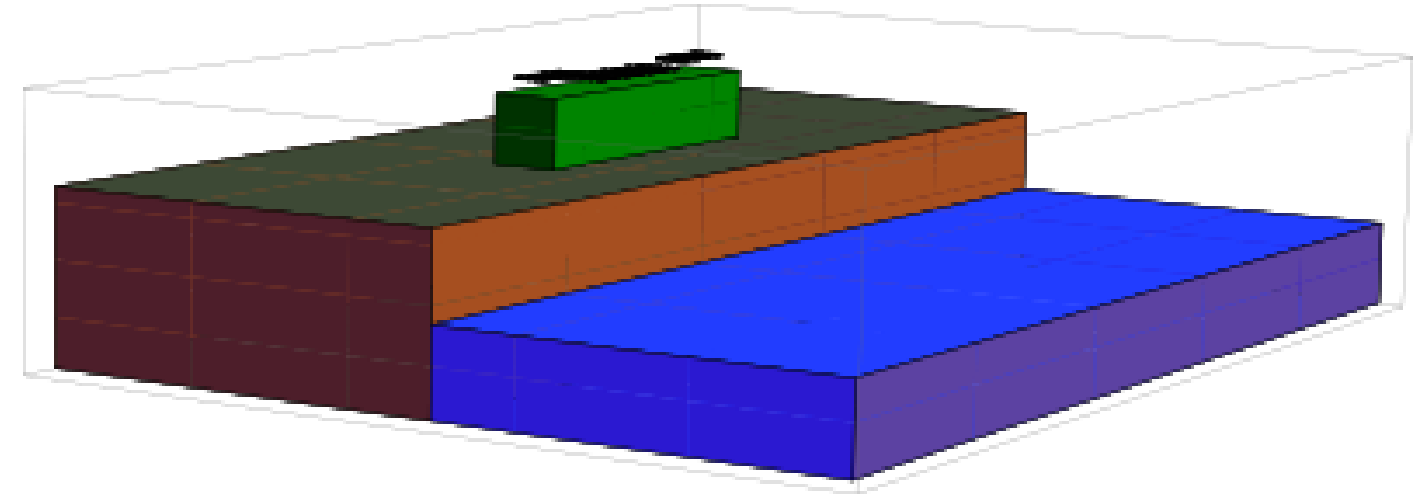

Figure 1-3. Scenario for Background Simulations

This model was used to calculate source detection scenarios consisting of a background and HEU source contained within an IMCC filled with some amount of uniform density wood cargo. The wood cargo was simulated with a mass density of 0.46 grams per cubic centimeter to approximate dry pine plywood. To construct realistic detection scenarios, several components were modeled independently and then reconstructed by later data manipulation. The terrestrial background (with and without the IMCC container and cargo present) and the source emissions from the HEU test source were modeled in separate MCNP runs and were used to construct permutations of this detection scenario.

\subsection{Soil Background}

We expect that an actual cargo inspection (using a real SBRD) will be preceded by a baseline measurement of the background without the presence of an IMCC. When an IMCC is being off-loaded, the measured background will be attenuated by the container and its cargo, which may not be known, and the measurement may include contributions from additional hidden sources.

Considering this, the terrestrial background was calculated with a series of MCNP models that included the SBRD and the 10 combinations of IMCC and cargo listed in Table 1-1. We have simulated and tallied count rates for every detector in the SBRD. Each spectrum was represented by $10243-\mathrm{keV}$ channels spanning the range from 0 to $3.076 \mathrm{MeV}$. We then normalize the rate to correspond to source counts per second (cps) per channel by adding a multiplicative cofactor into the MCNP input files. 
The spectra are in the 10 MCNP output files listed in Table 1-1. The maximum wood thickness considered (corresponding to $100 \%$ of the maximum) was $223 \mathrm{~cm}$. This corresponds to an areal density of $102.6 \mathrm{~g} / \mathrm{cm}^{2}$ for detectors immediately above the source, but up to $123 \mathrm{~g} / \mathrm{cm}^{2}$ for detectors near the edge of the region used for algorithm evaluation (see Fig. 1-7).

Table 1-1. Soil Background Simulation Output Files

\begin{tabular}{|l|l|}
\hline \multicolumn{1}{|c|}{ Description } & \multicolumn{1}{c|}{ File Name } \\
\hline No container & BG-75D-NC-Y6Z0.j.out \\
\hline Empty container & BG-75D-EC-Y6Z0.j.out \\
\hline $5 \%$ of maximum wood thickness & BG-75D-5-Y6Z0.j.out \\
\hline $10 \%$ of maximum wood thickness & BG-75D-10-Y6Z0.j.out \\
\hline $15 \%$ of maximum wood thickness & BG-75D-15-Y6Z0.j.out \\
\hline $20 \%$ of maximum wood thickness & BG-75D-20-Y6Z0.j.out \\
\hline $25 \%$ of maximum wood thickness & BG-75D-25-Y6Z0.j.out \\
\hline $50 \%$ of maximum wood thickness & BG-75D-50-Y6Z0.j.out \\
\hline $75 \%$ of maximum wood thickness & BG-75D-75-Y6Z0.j.out \\
\hline $100 \%$ of maximum wood thickness & BG-75D-100-Y6Z0.j.out \\
\hline
\end{tabular}

The calculated spectrum in the one center detector for each of the 10 soil-background configurations is shown in Figure 1-4. The top (dark blue) curve shows the calculated background spectrum for the case where no IMCC is present. The bottom curve shows the spectrum for the case of a container with a full load of wood. These simulations show the similarity of attenuated backgrounds over the range of cargo considered by this report. The normalization factor used in the MCNP output is $1.053 \times 10^{8}$, which is the emission rate in cps for the soil source. Background simulations included $2 \times 10^{10}$ random particle histories to establish high statistics.

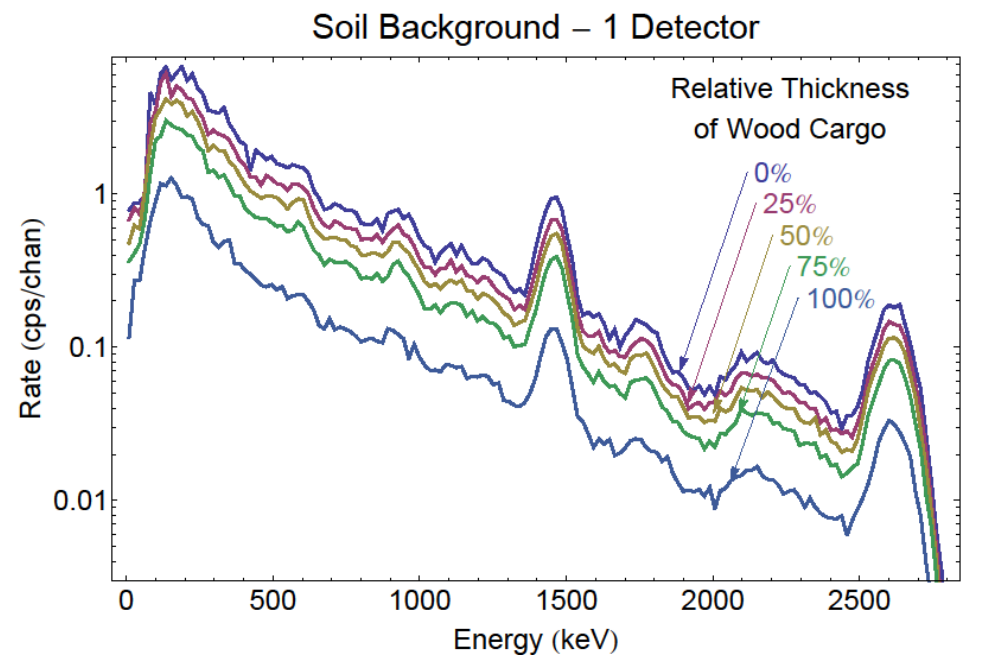

Figure 1-4. Soil Background Detected by SBRD Central Detector 


\subsection{HEU Source}

An HEU source was added to the MCNP model to represent a potential hidden source within the IMCC. The signal from the HEU source was calculated in with 10 levels of cargo. In all cases, the MCNP model for HEU used a point-source configuration for simplicity.

The spectrum emitted by the point source was the same as that emitted from the surface of a bare, spherical, HEU source [2] The total emission rate was chosen to match the rate at the surface of a 1-kg cylinder of HEU, with an equal diameter and height of $4.066 \mathrm{~cm}$. The point source was located where the center of the HEU cylinder would be if it were centered in the container and sitting on the container floor. The spectra are in the nine MCNP output files listed in the following table.

Table 1-2. HEU Source Simulation Output Files

\begin{tabular}{|l|l|}
\hline \multicolumn{1}{|c|}{ Description } & \multicolumn{1}{c|}{ File Name } \\
\hline Empty container & HEU-PS-75D-EC-Y6Z0.j.out \\
\hline $5 \%$ of maximum wood thickness & HEU-PS-75D-5-Y6Z0.j.out \\
\hline $10 \%$ of maximum wood thickness & HEU-PS-75D-10-Y6Z0.j.out \\
\hline $15 \%$ of maximum wood thickness & HEU-PS-75D-15-Y6Z0.j.out \\
\hline $20 \%$ of maximum wood thickness & HEU-PS-75D-20-Y6Z0.j.out \\
\hline $25 \%$ of maximum wood thickness & HEU-PS-75D-25-Y6Z0.j.out \\
\hline $50 \%$ of maximum wood thickness & HEU-PS-75D-50-Y6Z0.j.out \\
\hline $75 \%$ of maximum wood thickness & HEU-PS-75D-75-Y6Z0.j.out \\
\hline $100 \%$ of maximum wood thickness & HEU-PS-75D-100-Y6Z0.j.out \\
\hline
\end{tabular}

The normalization factor used in the MCNP output is $1.32 \times 10^{6}$, the emission rate in cps for the HEU source. As with the soil background simulations, all MCNP calculations were continued for $2 \times 10^{10}$ particle histories. The spectrum detected in the center detector for each of the nine cargo thicknesses is shown in Figure 1-5. The top curve is for an empty container and the bottom curve is for a container with a full load of wood. While the highest integrated count rate (with no cargo) is $722 \mathrm{cps}$ for the one central crystal, the corresponding count rate at $100 \%$ cargo fill is only $2.6 \mathrm{cps}$. This lowest rate is small enough that no detection is expected for a single HEU source.

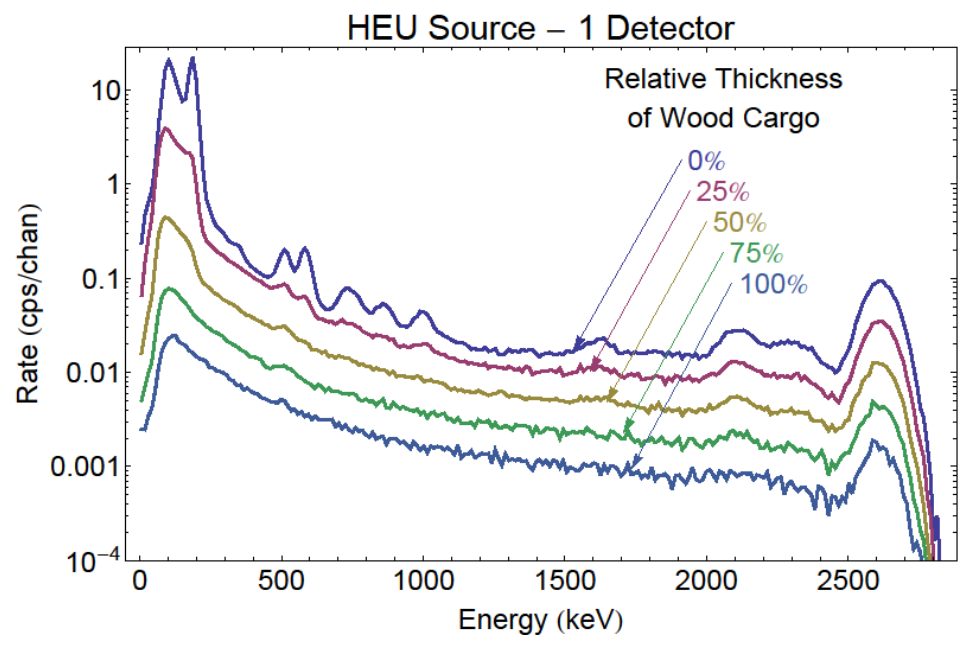

Figure 1-5. HEU Source Detected by SBRD Central Detector 
Each of the MCNP output files contains 75 output tallies, corresponding to the spectra at each of 20 detector crystal locations at one end, followed by the 35 central locations and the 20 locations at the other end (see Figure 1-1). A subset of these tallies was summed to generate optimal sensitivity to a single source, as described in section 1.5.

\subsection{Data Preparation}

With data sets corresponding to the scenarios described above (and with high statistics simulating very long counting times), a set of data was constructed to simulate the count rates that a real detector would see during field operations. In a real measurement of a cargo container, available data will include a background (taken beforehand, without any cargo container or additional source present) and the cargo container measurement. The latter will include the background attenuated by the cargo and container as well as possible contributions from hidden sources. For all simulations of real measurements prepared from this data,we choose a measurement time of 60 seconds to represent the approximate time taken in cargo loading.

We construct detector observations in the ASC file format by first declaring a scenario with a source emission rate (defined as a simple multiplication of the emissions from the HEU test source) and a volume of cargo (up to 100\%). The background (with no cargo container) was scaled to a 60 -second observation and retained for comparison. The background attenuated by the IMCC and cargo, and the HEU source tallies were scaled and added together to represent the measurement taken during crane loading. Many statistical "realizations" of those results were made by adding noise corresponding to the appropriate Poisson variation of the counts in each energy bin. In this way, many realizations were simulated of each combination of cargo depth and source strength, between 0 and around 160,000 percent of a single test source's emissions. We choose this range to allow for source detection by at least some algorithms, even with deep cargo shielding.

\subsection{Analysis Methodology}

In order to apply algorithms to the data from the ASC files, thresholds will be necessary (the point at which the result of an algorithm can be used to signal detection of a source). For this work, the effects of naturally occurring radioactive material (NORM) were not considered and the statistical limit was used to evaluate the performance of each algorithm. The statistical limit is gained by applying each algorithm to the set of scenarios (with any cargo depth including no cargo or container) containing no additional source, and setting the threshold high enough that only an allowable number of false alarms would be seen (a false alarm probability of 0.001 was considered acceptable). The lowest threshold allowing this false alarm probability was used as the detection threshold for that algorithm.

To simulate optimal use of the detector array, the optimal number of detectors to be used (i.e., summed together in a single measurement) has been investigated. In general, using only one detector element (and applying the algorithm in question only to the single crystal with the highest count rate) will lose resolving power of the instrument, while always summing every detector together will include too much background and reduce sensitivity. Therefore, we seek some "optimal number" between these two extremes.

For this study, we assume that the detector most closely approximating the source's location in the cargo is known from inspection of the relative count rates in each crystal. Then, we define the optimal number 
of detectors to sum together as the number which yields the greatest signal-to-noise ratio for the sum. The signal used for this optimization is the rate from the HEU source at the bottom of the cargo container with a full load of wood cargo. The noise is the square root of the total background rate with the same full load of wood cargo. The signal-to-noise ratio is plotted in Figure 1-6 as a function of the number of summed detectors starting from the center detector. The inset shows a detail of the curve near its maximum, the section corresponding to the 15 to 25 nearest detectors. The best signal-to-noise ratio comes from using 21 central detectors. These are shown in red in Figure 1-7. These 21 central detectors are the middle $5 \times 5$ array of detectors minus the four corner detectors.

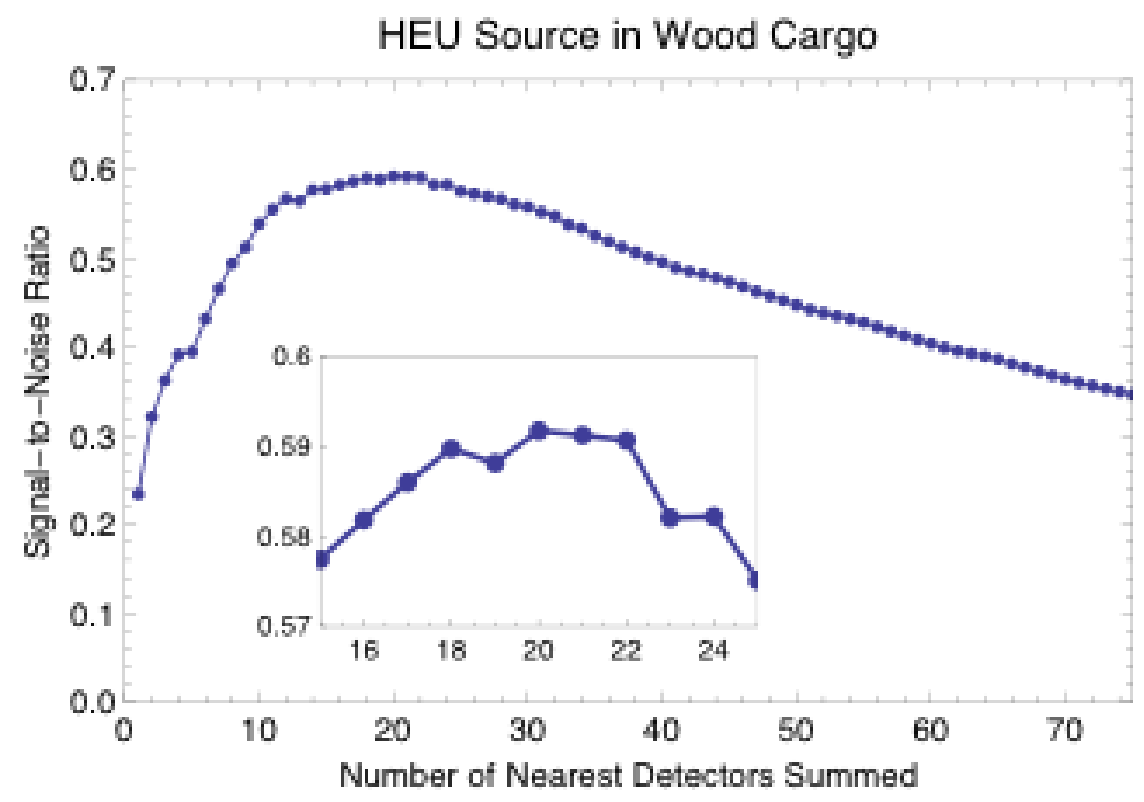

Figure 1-6. Signal to Noise Ratio

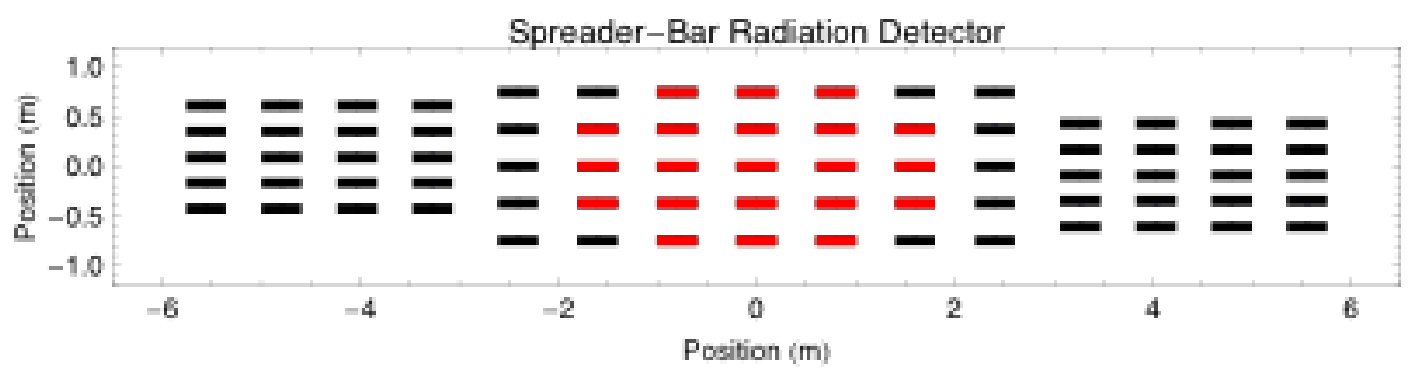

Figure 1-7. Optimal Detectors to Include in Sum

Each MCNP output file contains 75 output sections (tallies), corresponding to the 20 detector locations at one end of the spreader bar, the 35 detector locations in the middle part of the spreader bar, followed by 20 detector locations at the other end of the spreader bar (all the locations depicted in Figure 1-7). The center detector corresponds to output sections number 38 out of $75 \mathrm{MCNP}$ output tallies. The optimal group of 21 detectors corresponds to output tally sections 27-29, 31-45, and 47-49. These 21 sections are summed together to produce results. An important caveat to this work is that only a central location was chosen for the source, and other locations have not been considered. Further work will be necessary to explore the interplay of source location, source detection and detector subsets, and develop an algorithm which automatically chooses the optimal subset of detectors to sum. 


\subsection{Data for Analysis by Algorithms}

Several data sets were produced from these source/background simulations. Statistical realizations were made of each cargo and source permutation, including no source at all. Two hundred realizations of each permutation were made by adding noise to an original with high statistics. Additionally, realizations of the scenario containing no IMCC or cargo were constructed for comparison. These data were used to make a set of ASC files, with every depth and source emission rate for 60 seconds, which were spotchecked for correctness. 


\section{Algorithms}

Several algorithms were considered for comparison and are briefly described below, along with a short explanation of the figures of merit they return. In all cases, the figure of merit is compared with a detection threshold to produce alarms.

\subsection{Gross Counting}

The gross-counting algorithm is one of the simplest methods of source detection and is used in many deployed detector schemes. In this algorithm, the number of counts above the background is computed and compared with a threshold. The figure of merit $\mathrm{F}$ is generally calculated:

$$
\begin{aligned}
& \mathscr{A} \boldsymbol{A} \boldsymbol{A} \text { or } \\
& F=(C-B) / \sqrt{B}
\end{aligned}
$$

where $C$ is the total counts and $B$ is the background counts. Gross counting is thought to be an effective "baseline" solution as its performance will not be significantly changed by anything other than attenuation by cargo materials.

\subsection{Energy Distance}

Previous work has shown improved results in some cases of source detection with the use of "energy distance" anomaly detection algorithms [3]. In this approach, the available spectrum is first binned into coarse energy bins (eight bins are used for this investigation). These bins are treated as an eightdimensional vector and normalized. Then the "opening angle" between background and source spectra is found by taking the dot product between them. This opening angle is the figure of merit returned from this algorithm.

\subsection{Peak Finding}

The peaks present in the spectroscopic data can be "picked out" with a variety of methods and then compared with a catalog of known source emission lines to produce a list of the isotopes present in the measured spectrum. The peak-finding algorithm used for this work first subtracts background and then takes the convolution integral of the data with a Gaussian peak of width matching the detector resolution. The resulting function over energy is compared with a threshold to generate peaks and these peaks are compared with a limited catalog of sources to determine source detection. In this case, a detection of HEU (that is, a detection of the relevant emission lines from U-235) is considered to constitute a positive detection, while any other isotopes are ignored.

\subsection{GADRAS}

In the template-matching technique used by the Gamma Detector Response and Analysis Software (GADRAS), the spectrum to be analyzed is compared with a library of templates. When a "good" match 
is found, the spectrum can be identified as that of the isotope used to generate the template. The template library contains the measured or calculated spectra of most isotopes as well as some background spectra.

Template matching is well suited to the identification of unshielded radiation sources; multiple regressions can also be applied to identify combinations of radionuclides. Template matching can be performed quickly with limited computational resources, a desirable feature for use in handheld radioactive isotope identifiers. The GADRAS template-matching code was used in this work for identification of the HEU source [4]. In this case, the presence of uranium in the list of detected sources is used as a positive indicator of detection, meaning that the figure of merit for this use of GADRAS is effectively binary. While false alarms may be present in the form of declarations of other sources not present in the data, there is no trivial way to set a threshold on detection using the GADRAS algorithm, and the probability of detection is therefore reported irrespective of false detections.

\subsection{Limited GADRAS}

GADRAS is employed in one other fashion in this work. In this technique, only the natural isotopes expected in background were included in the GADRAS template library. The presence of an additional source will tend to make the "goodness of fit" progressively worse with intensity. Thus, the goodness of fit $\left(\mathrm{Chi}^{2}\right)$ is used as the figure of merit for this use of GADRAS, rendering it an anomaly detecting algorithm.

\subsection{Production of Results}

We apply each algorithm to all the background files and produce data digests containing values of cargo depth, iteration number, and figure of merit. We also apply the algorithms to our source data set to produce another file with the values of cargo depth, source rate, iteration number, and figure of merit. We declare the acceptable false alarm probability to be about 1/1000 and determine an appropriate alarm threshold that gives this for each algorithm, for any part of the data set not containing any source term (i.e., taking the statistical limit of algorithm performance).

These results are post-processed by comparing the alarm algorithms for each algorithm to the figures of merit for all the source runs. The number of source runs exceeding the thresholds are combined to produce plots of source emissions versus probability of detection for each algorithm and depth. After this, for each cargo depth, the source emission rate necessary to produce a probability of detection of $95 \%$ is found by interpolation of the data (or by extrapolation where necessary) and a plot of minimum detectable source amount versus cargo depth was produced for each algorithm. 


\section{Results}

We find and report the results of the preceding analyses by first evaluating the Figures of Merit (FoM) for each algorithm and then using these to calculate the Minimum Detectable Amount (MDA) of the HEU source for each possible depth of cargo within the IMCC. The figures of merit are plotted for each algorithm (see Figs. 3-1, 3-2). In these figures, the FoM is scaled by the detection threshold of each algorithm (such that values above 1 would indicate detection), to show clearly the region in which a detection would be made. .

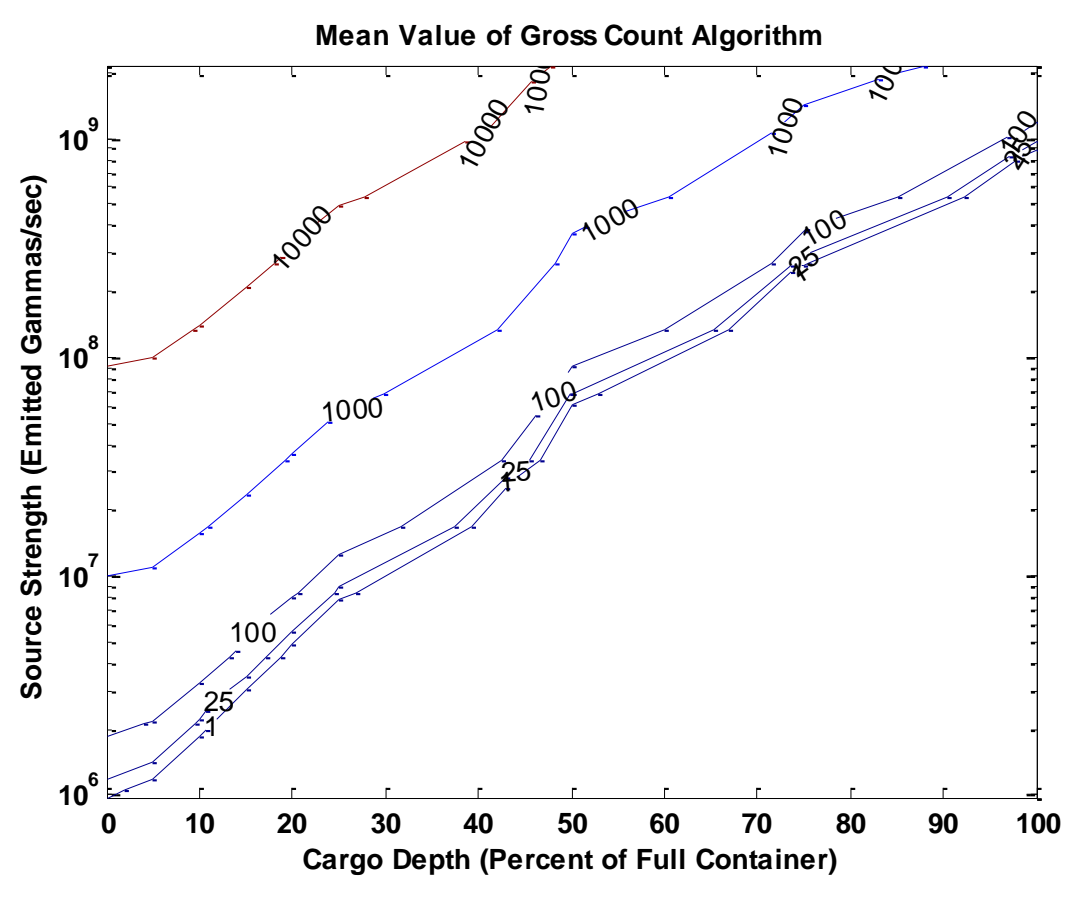

Figure 3-1. Results from the Gross-Count Algorithm 


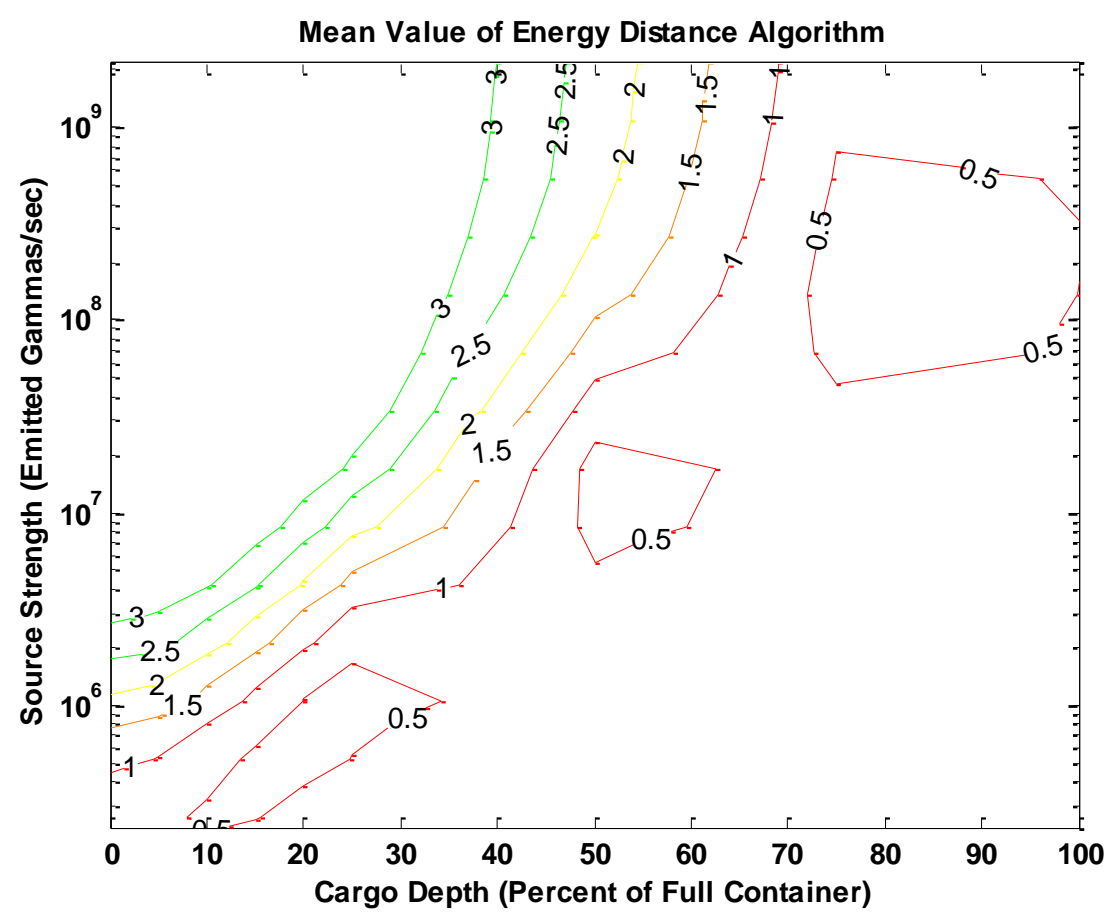

Figure 3-2. Results from the Energy Distance Algorithm

These data are processed again, to find the MDA for each cargo depth by determining the amount at which the detection probability exceeds $95 \%$. The data are reanalyzed to produce the PD for each source strength and cargo depth, given the thresholds used above. The source strength at which PD $=0.95$ for each cargo depth is approximated by interpolating these data (or extrapolating in the case that even the strongest sources investigated were not detected). The resultant minimum detectable amounts are shown in Figure 3-3, for increasing cargo depth. 


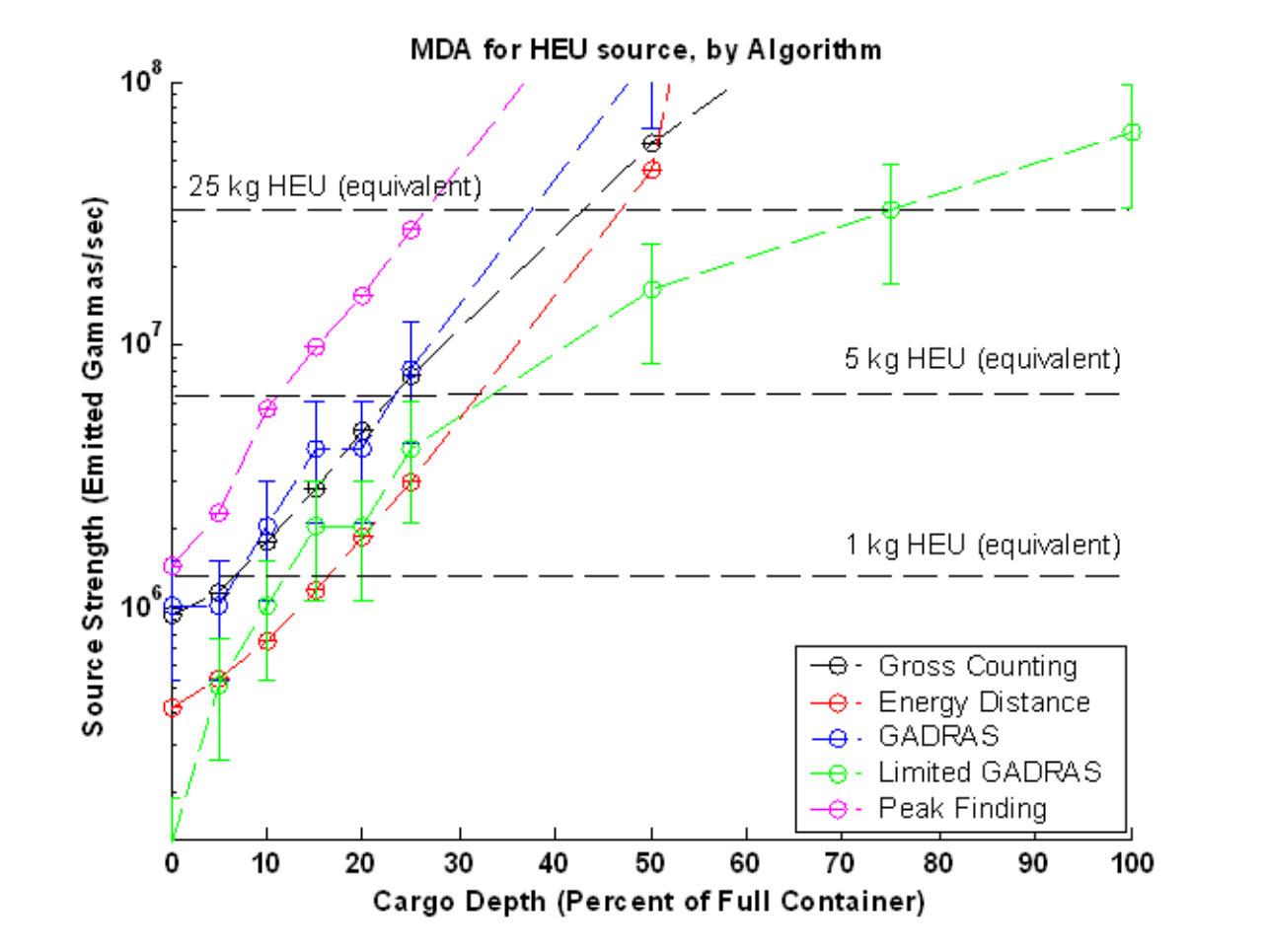

Figure 3-3. Minimum Detectable Amounts for HEU Source

Error bars are determined by calculating the variance of the Probability of Detection as a function of source strength, and then inverting this to estimate the variance of source strength at $P D=0.95$. In Figure 3-3, error bars for the gross-counting, peak-finding, and energy distance metrics are smaller than their respective symbols in these plots, due to the relatively low variation in the FOM returned from these algorithms when only statistical variations of scenarios are used as input. Results from these findings show a range of performance within the choices of algorithm. The performance of GADRAS is similar to, or slightly better than gross counting at the limit of light shielding. This is as expected for cases in which additional NORM sources, which tend to confound non-energy-based algorithms, are not considered. Also, algorithms which tend to depend on the measured spectral shape or photopeaks in the HEU spectrum (GADRAS, energy distance, and peak finding) have a higher slope, showing the degradation of performance with increased shielding. In general, energy-ratio or energy-binned anomaly detecting schemes may provide the "best case" for detection, superior to the gross-counting result for cargo up to about 50\% of maximum (around $111 \mathrm{~cm}$ ) and the best overall out to around 30\% (around 67 $\mathrm{cm}$ ). Additionally, the limited GADRAS implementation (using the algorithm as an anomaly detector) significantly outperformed the others in the limit of very deep cargo. 


\section{Discussion and Conclusions}

Several results are of special note here. Firstly, the relatively poor performance of the peak finding algorithm may not be an indictment of its performance in all cases. Due to volatility of the algorithm, a few "high confidence" detections were made in scenarios with no actual additional source. This necessitated a high threshold to maintain the low false alarm probability required in this study. It is possible that further algorithm tuning might alleviate some of this issue.

As would be expected, algorithms that rely on specifics of peak structure of the spectra tend to degrade more quickly as shielding becomes large. However, these sorts of algorithms perform well with light shielding and showed reasonable capability to identify sources at these levels. Again, NORM sources were not included here, which tend to increase the threshold necessary on gross-counting algorithms.

Perhaps the most surprising and encouraging result of this study was the performance of the limited GADRAS implementation. This use of GADRAS far outperformed the other algorithms in deeply shielded situations, suggesting that the use of natural isotopes as templates to represent background and shielded background is very successful. Also, the addition of NORM is not expected to greatly affect the performance of this algorithm, as the likely NORM sources are contained within the set of templates already in use. However, none of the algorithms provided positive detection with full cargo unless the source intensity was above 25 times the test source. Additionally, the similar performance of the Energy Distance algorithm below a $5 \mathrm{~kg}$ equivalent source highly indicate its use in many scenarios.

An important caveat to this work is that the choice of algorithms made herein is not exhaustive and is meant instead to provide benchmarks and relative comparison of known algorithms. Further work will refine these concepts and determine implementation specifics to maximize the performance across a range of sources and scenarios.

\subsection{Outlook for Using Real Data}

Several simplifications were made over the course of this work. Notably, because the background and source measurements were simulated, they provide only statistical variances. This leads to a artificially low uncertainty to the minimum detectable quantities of a source for a given scenario, as opposed to the systematic uncertainties expected from real source placement, deviation in individual detector efficiencies and calibration, and so on. For this and other reasons, it is necessary to obtain and use some real repeated measurements of background taken from a similar detector.

Also, only one scenario (an HEU cylinder near the bottom of an IMCC full of wood cargo) was represented by this work, and additional sources and cargos would be of interest. The real time-variation of background was not simulated and was instead averaged over. All of the results from this work would be enhanced by the investigation of real data including fielded radiation sources.

\subsection{Future Avenues for Investigation}

The current results are encouraging regarding the performance of anomaly detection algorithms specifically, as they show substantial improvement over the standard gross counting and template matching approaches. Several outstanding issues are obvious future choices for investigation and are expected to be of great use to the deployment of a real detector. 
The assumption about the subset of detectors for best source detection will be challenged and explored. We have currently established the "best subset" for a source near the bottom of a container, but have not established the variation in this subset as the source location changes or the performance of a real selection criterion as the source strength goes toward zero. These should be explored, since any real detector will need such an applicable criterion that maximizes real detection capability without any prior knowledge about source presence or location.

Most importantly, in our current analyses, the limited GADRAS and energy anomaly algorithms seem to perform well. It is expected that some combination of these concepts will yield the very best result. To that end, it makes sense to merge these concepts and establish one, optimal recommended algorithm. In this effort, the concept of the best background components to use will be considered, as well as binning issues and the best detection metric. 


\section{References}

1. X-5 Monte Carlo Team. MCNP-A general Monte Carlo n-particle transport code, version 5.Vol. I, LA-UR-03-1987. Los Alamos National Laboratory, Los Alamos, New Mexico. 2003.

2. Padmini Sokkappa, Charles Guenther, Bert Pohl, Shiva Sitaraman, Eric Smith, Robert Hillaire, "Benchmark Sources for Radiation Detection Architecture Evaluation (revision 2).", Lawrence Livermore National Laboratory UCRL-TR- 218277-REV-2.

3. S. M. Robinson, S. E. Bender, E. L. Flumerfelt, C. A. LoPresti, and M. L. Woodring. "Time Series Evaluation of Radiation Portal Monitor Data for Point Source Discrimination." Journal of Radioanalytical and Nuclear Chemistry (DOI: 10.1007/s10967-009-0176-6).

4. D. J. Mitchell. DHSIsotopeID and Full-Spectrum Analysis Methods in GADRAS. SAND 20092544P. Sandia National Laboratories, Albuquerque, New Mexico. 2009. 


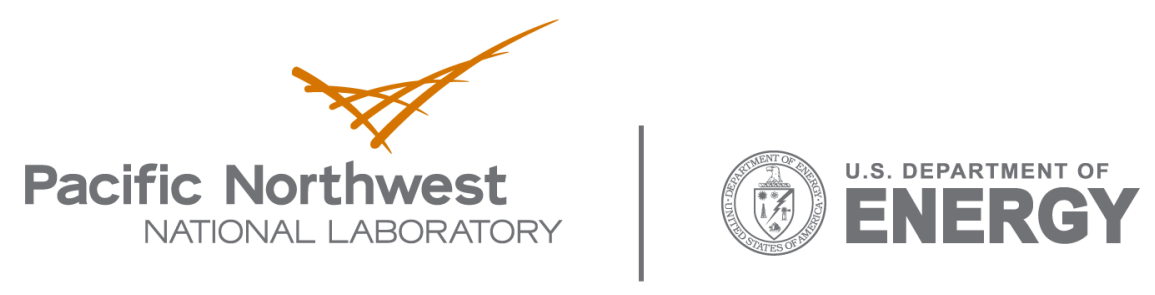

902 Battelle Boulevard

P.O. Box 999

Richland, WA 99352

1-888-375-PNNL (7665)

www.pnl.gov 